TWO CASES OF

\title{
FRACTURE OF THE THIGH-BONE
}

TAKING PLACE WITHOUT ANY VIOLENCE, '

$$
\text { IN WHICH }
$$

A DISEASED STATE OF THE BONES APPEARS TO HAVE BEEN THE PREDISPOSING CAUSE OF FRACTURE,

AND CONCURRING WITH

\section{CANCER IN THE BREASTS}

IN BOTH PATIENTS.

BY THOMAS SALTER, ESQ. F.L.S.

SURGEON, POOLE, DORSET.

Read December 23rd, 1828.

$\mathrm{ON}_{\mathrm{N}}$ the 22nd of July, 1823, Mrs. Lock, aged 82 years, who had been many years a respectable nurse, whilst standing at her drawers to take out some linen, felt the right thigh suddenly break, and she fell upon the floor; some persons who were in an adjoining room immediately came to her assistance, and I was sent for ; I found her in a sitting posture in the same place, and just as she fell. On examination, the thigh-bone was found to be broken a short distance below the trochanters; the movements consequent on the examina- 
tion produced no crepitus; but a good deal of spasm of the muscles was observed, occasioning considerable distortion of the limb. The case was treated in the usual way, and at the end of three months the bone had acquired some firmness. For several months previous to the accident, she had with little exception constant and very severe pain in the thigh, more especially at the part where the fracture took place. The disease giving rise to this very severe pain had rendered her so lame that she was unable to leave her room; but with the aid of a stick she could just move about the chamber; the pain left her as soon as the accident happened, and did not afterwards return. This poor woman had been long afflicted with a cancerous ulceration of the mamma; the ulcerative process increased after the fracture of the thigh, which, together with the confinement to bed that the broken bone necessarily occasioned, added to her great age, produced such a waste of the vital energies, that she gradually sunk, and died December 25th, 1823 .

The friends of the patient would not allow of an inspection of the thigh-bone after death, but from an examination of the limb, it was found to be so flexible, that I am disposed to believe no ossific union had occurred. In the following case (its prototype) I had full permission for a post mortem examination, and the account subjoined of the 
dissection will I think be considered as a suffcient illustration of the disease in both instances.

July 28th, 1824. I received a message to attend on Mrs. Pringle of this town, who was stated to have broken her thigh. The following is a history of the accident, and of the previous symptoms. This patient, who is 56 years of age, had, for about five months prior to the fracture, laboured under violent pain of the right thigh, extending from the hip-joint to the knee, which seemed deep seated as if in the bone. A little above the patella, in front of the limb, there has been for some time a slight tumefaction, which appears to arise from a thickening of the periosteum ; it is tender on pressure, indeed the whole member is morbidly sensible to external impressions. The pain has been severe at all times, but more so at night ; it had produced great lameness; the muscles of the thigh are much shrunk. For many years she had complained of slight rheumatic pains in the affected thigh, and for some time after this attack commenced, she supposed it to be of the same nature, and she attributed its subsequent increased severity to inadvertently striking her foot, about four months ago, against one of the steps of the stairs; but it would appear more probable that this accident arose from inattention and a weakened state of the muscles produced by previous disease, so that, in ascending the stairs, 
the leg was not raised sufficiently high; however since this trifling occurrence, her sufferings have been uniformly more violent. A variety of means were employed for the relief of the patient, but these failing of their expected effects, she was recommended to try change of air. 'In the attempt to get into the cart which was to convey her into the country, the accident happened; she had been lifted up to the front of the vehicle, on that part where the driver's feet are usually placed, and in the act of raising the affected limb over the front to step in, the thigh-bone broke with a noise distinctly audible to the persons about her; she was again immediately taken into the house and placed in bed; the fracture was about three inches below the trochanter major; there being little or no spasm of the muscles, the extension and proper adjustment of the fracture was readily effected. The thigh was placed on an inclined plane made of pillows, and the usual bandages and splints applied; no crepitus was discovered on reducing the fracture. Since the accident she has felt no pain, unless pressure was made on any part of the thigh. For several years the patient had a scirrhus tumor of the left breast.

In October 1823, she was received into Guy's Hospital, and on the 10th of that month Sir A. Cooper removed the disease ; the wound healed readily; but the cicatrix, about six months since, became affected with cancerous ulceration. The discharge and pain attending which considerably 
impaired the general health. The patient lingered till October 19th, 1824, without any improvement in the state of the thigh, excepting the absence of pain. On the 20th I removed the thigh-bone, and brought it home for examination; but previous to its removal, the affected limb was observed to be considerably shorter than the other, flexible at its middle, and a good deal distorted from a projection just below the trochanter major. The muscles of the thigh were pale and shrunk; a bloody fluid escaped from the capsular ligament of the knee-joint, and two or three small clots of pure blood were in the articular cavity. On removing the patella, an ulcer about the size of the little finger nail was discovered in the upper and external part of the articulatory surface of the bone.

Circumstances rendered it necessary that the: bone should be removed as quickly as possible, and in my haste to separate the head from the acetabulum the neck was accidently cut through by the scalpel, and the head of the bone left in the cotyloid cavity; in afterwards detaching the head, the ligamentum teres was torn through by the finger. The head of the bone had lost its wonted smooth, cartilaginous polish, being rough and somewhat soft near its centre. . The thigh-bone was remarkably soft throughout its whole length, and the knife could be pushed through it at any part, but at its middle it was most conspicuously deficient in earthy matter; at about three inches from either: extremity it was so flexible that it could 
be bent in any direction. It was at the upper part of this portion where the fracture took place, but it would be difficult from the preparation to point out the precise spot; in all probability there was no entire separation, such as occurs in common fractures. The distortion of the limb did not, it is clear, arise from any overlapping, but from 2 bending of the bone from a contraction of the. muscles. The muscles in immediate contact with the trochanters and the upper half of the limb were blended together into a uniform mass of a pale red colour, firm and semicartilaginous; having bony spicula thickly dispersed through them, and puriform matter slightly tinged with blood issuing from the cut surfaces. The common integuments had suffered no change. Corresponding to the swelling : which had been ob: served on the front of the bone above the patella, the tendon of the cruralis was much thickened, and altered in texture, and a considerable quantity of pus came from under it; and when the tendon of this muscle was detached from the periosteum, the latter membrane was found greatly thickened; and readily separated from the bone. The table of the thigh-bone at this part was discovered to be almost entirely absorbed quite down to the condyles; the medullary cavity was filled with a bloody pultaceous substance; indeed so great was the disorganization that $I$ find it difficult to give such a description of it as shall convey to the reader an accurate idea of the change of struc- 
ture the bone had undergone; but I trust the accompanying drawings*, made by my intelligent pupil, Mr. Bullar, will in a measure supply what may be found deficient in description. They are representations of rather more than the lower third of the thigh-bone, passing through and including the condyles. One of the portions is drawn as it appeared immediately as the section had been made; the other, from the dried preparation after a tedious maceration by which all the soft parts were decomposed and separated. The latter preparation is only of the trifling weight of four drachms and a half troy.

The foregoing cases have so many points of analogy, that we are naturally led to suppose the predisposing cause of fracture to have been the same in both cases. Both the patients laboured under cancer of the breast; both suffered much from previous pain and lameness; and in both instances also, the accidents occurred without the agency of the usual external causes; indeed the only circumstance in which they do not entirely accord is the age of the patients. However, it must be remembered that a numerical disparity in years is not always a correct measure of the constitutional differences of individuals. The bones of elderly people sometimes undergo a change of structure by which they become soft- 
ened in their texture, and rendered liable to fracture by the operation of trifling causes. In his splendid work on "Dislocations and Fractures of the Joints," Sir Astley Cooper makes the following observation on the state of the bones of old people: " that the regular decay of nature which is called old age, is attended with changes which are easily detected in the dead body; and one of the principal of these is found in the bones; for they become thin in their shell and spongy in their texture." So easily are bones broken that have undergone this change, that the shaft of a thigh-bone, the neck of which was shortened, was broken by $\mathrm{Mr}$. South in his attempt to remove it from a body in the dissecting-room at St. Thomas's Hospital *. But it has not been observed, that the changes here alluded to are accompanied with either pain or lameness, which form two principal features in the cases here submitted to the profession, besides which, one of the patients had not arrived at an advanced age. I therefore infer, the disease with which these two persons were affected, to be essentially different from the change in the shaft and upper part of the thighbone, incident to advanced age. Mr. Samuel Cooper is the only surgical author to which I have access, who has taken any notice of this disease. In his "First Lines of Surgery," he observes, "that Louis and Saviard mention cases

* Sir A. Cooper on Dislocations and Fractures of the Joints. vol. Xv. 
in which women affected with old ulcerated cancers have fractured their bones in merely executing the natural and ordinary motions of the body, as merely changing their positions in bed. No mention is however made by Mr. Cooper of pain or lameness preceding these fractures; and as $I$ have no opportunity to consult the authors by whom the observations were made, I cannot determine whether they were really affected by any premonitory symptoms. However, it is not impossible that these symptoms may have existed, though they may not have been considered by the authors of sufficient importance to require to be recorded. If the experience of other surgeons accord with Louis and Saviard, it would seem to establish a connection, nearly allied to cause and effect, between carcinoma and this peculiar kind of fracture. Whether the disease in the bone be in itself actually of a carcinomatous nature, or of some other kind, excited by the cancerous virus in the habit, it is perhaps difficult to determine. If, however, a more enlarged experience should prove, that the two diseases have no connection, the cases mentioned by Louis and Saviard, as well as those above recited, must be allowed to constitute singular and remarkable coincidences. 

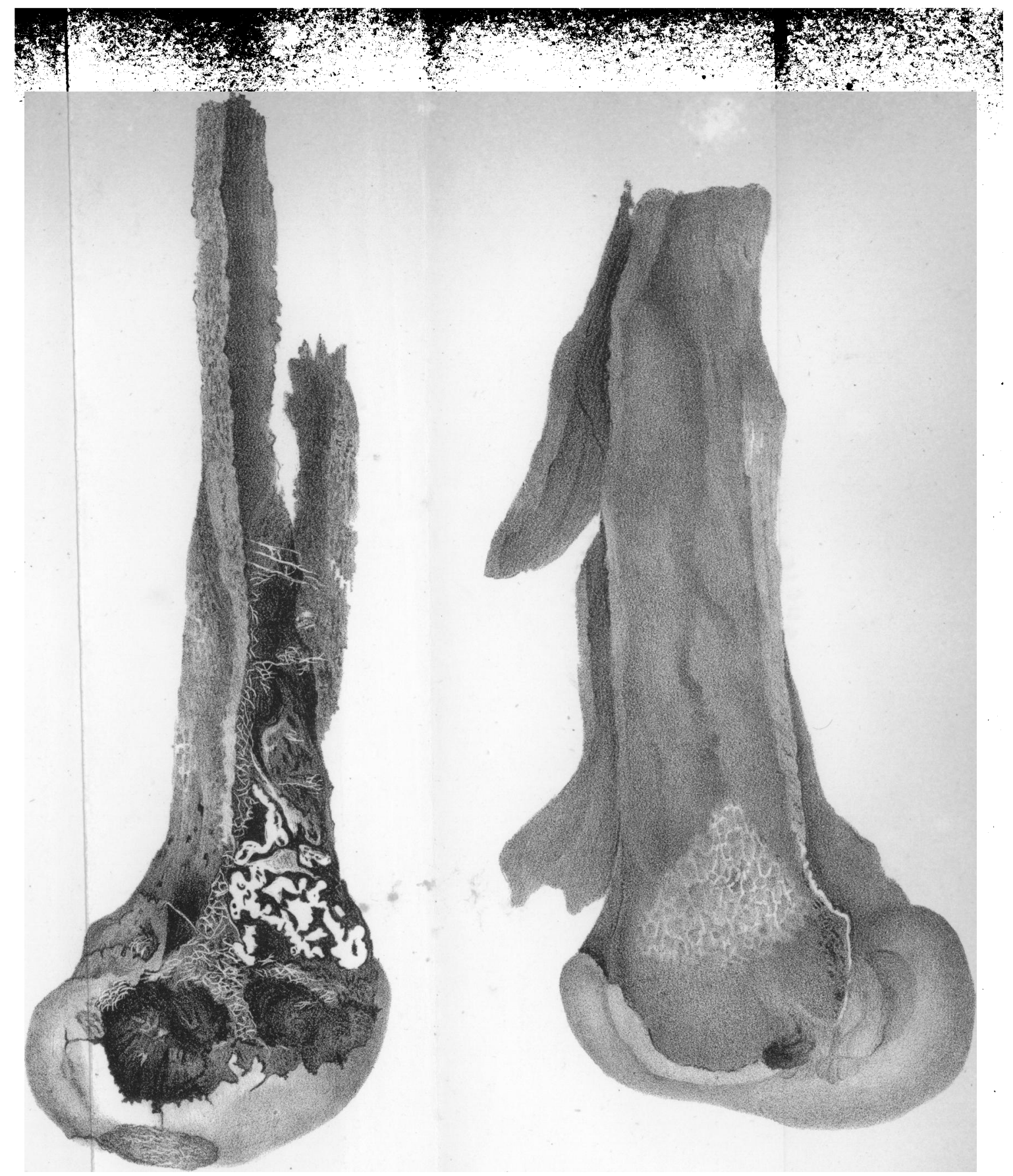